\title{
ULTRAFAST CARRIER TRAPPING AND HIGH RESISTIVITY OF MeV ENERGY ION IMPLANTED GaAs
}

\author{
K.P. Korona, J. Jasiński, A. KurPiewski, M. KamińsKa \\ Institute of Experimental Physics, Warsaw University \\ Hoża 69, 00-681 Warsaw, Poland \\ C. JAGADISH, H.H. TAN
}

Department of Electronic Materials Engineering, Australian National University ACT 0200 Canberra, Australia

\section{A. Krotkus and S. Marcinkevicius}

Semiconductor Physics Institute, A. Gostauto 11, 2600 Vilnius, Lithuania

Semi-insulating GaAs wafers were implanted with $\mathrm{MeV} \mathrm{As}, \mathrm{Ga}, \mathrm{O}$ or $\mathrm{Si}$ ions at doses ranging from $1 \times 10^{14}$ to $5 \times 10^{16} \mathrm{~cm}^{-2}$. Their structural properties were studied by electron microscopy and the Rutherford backscattering-channeling. Time resolved photoluminescence, electrical conductivity and the Hall effect were used to determine carrier lifetime and electrical properties of the material. Annealing of the samples at $600^{\circ} \mathrm{C}$ led to the recovery of transport in conduction band. The As, Ga and $\mathrm{O}$ implanted samples became semi-insulating, while the Si implanted samples were $n$-type. Carrier trapping times were short, shorter than 1 ps for the highest dose used. Models explaining the fast photocarrier decay are discussed.

PACS numbers: $61.72 . \mathrm{Vv}, 72.20 . \mathrm{Jv}, 72.80 . \mathrm{Ey}$

In the last few years there has been great interest in low temperature (LT) GaAs, highly non-stoichiometric As-rich material, because of its application in fast optoelectronic devices, and interesting physics behind its useful properties $[1,2]$. Successful attempts have also been made to create an alternative non-stoichiometric material by As implantation, and similar performance to LT GaAs has been achieved [3]. In this paper we show that GaAs implanted with different $\mathrm{MeV}$ ions at high doses and subsequently annealed, exhibits picosecond lifetime of photocarriers and relatively high conduction band mobility, all characteristics necessary for good performance in fast optoelectronic devices.

Semi-insulating (100) GaAs wafers were implanted with $\mathrm{As}, \mathrm{Ga}, \mathrm{O}$ or Si ions at doses ranging from $1 \times 10^{14}$ to $5 \times 10^{16} \mathrm{~cm}^{-2}$ using ANU Tandem accelerator. 
The implant energies were from 1 to $2 \mathrm{MeV}$ and were chosen such that all the ions would have approximately the same projected range. After the implantation the samples were annealed at temperatures from $400^{\circ} \mathrm{C}$ up to $800^{\circ} \mathrm{C}$ under an arsine ambient in a metalorganic chemical vapor deposition (MOCVD) reactor to prevent loss of arsenic from the surface.

The structural properties of implanted GaAs were studied by the Rutherford backscattering spectroscopy-channeling (RBS-C) and by transmission electron microscopy (TEM). In all cases, the RBS-C studies indicated the presence of postimplantation damage and showed that annealing leads to its substantial recovery. However, in the annealed samples an increase in dechanneling yield was observed as compared to the unimplanted samples, indicating that some defects still exist. It was also observed that dechanneling yield increased with the ion dose suggesting higher residual defect concentration for higher dose used. TEM images showed that during implantation a buried layer of about $1 \mu \mathrm{m}$ thickness was amorphized. However, after annealing at $600^{\circ} \mathrm{C}$ the amorphous layer recrystallized. The most interesting layer, $1 \mu \mathrm{m}$ below surface, was free of precipitates.

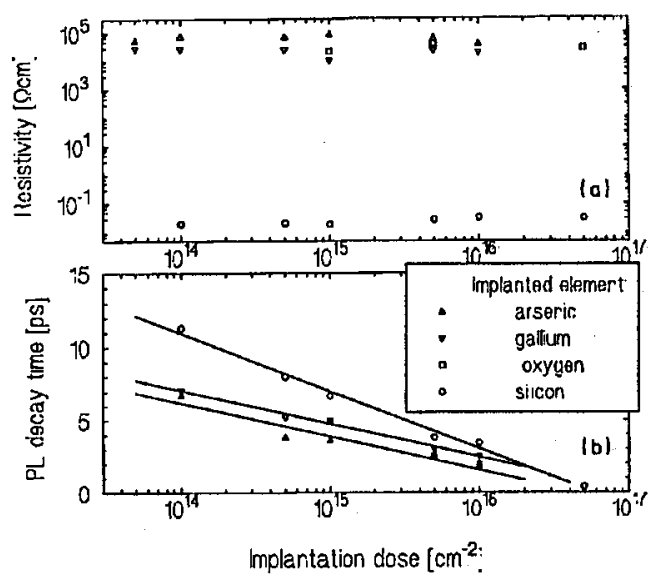

Fig. 1. The close dependence of (a) resistivity and (b) PL decay times, of GaAs implanted with $\mathrm{O}, \mathrm{As}, \mathrm{Ga}$ and $\mathrm{Si}$ ions and annealed at $600^{\circ} \mathrm{C}$.

Electrical characteristics of the samples were made using the Van der Pauw configuration. Typical value of applied current was a few $\mathrm{nA}$. It was observed that hopping conductivity which dominated in as-implanted samples vanished after annealing. In the samples annealed at $600^{\circ} \mathrm{C}$, an electron mobility from 1000 to $2000 \mathrm{~cm}^{2} /(\mathrm{V} \mathrm{s})$ was observed. For the calculation of resistivity and concentration a layer thickness of $1 \mu \mathrm{m}$ was assumed. The results showed that samples implanted with $\mathrm{As}, \mathrm{Ga}$ and $\mathrm{O}$ and annealed at $600^{\circ} \mathrm{C}$ had very high resistivity typical of semi-insulating material (Fig. 1a). Their electron concentration was between $10^{10}$ and $10^{11} \mathrm{~cm}^{-3}$ and activation energy of conductivity was $0.68 \div 0.02 \mathrm{eV}$. On the other hand, the samples implanted with $\mathrm{Si}$ had electron concentration $2 \times 10^{17} \mathrm{~cm}^{-3}$ for lower doses down to $1 \times 10^{17} \mathrm{~cm}^{-3}$ for higher doses which sug- 
gested that implantation above $10^{15} \mathrm{~cm}^{-2}$ led rather to increase in compensation ratio than to increase in electron concentration. Electron mobility of these samples was about $1800 \mathrm{~cm}^{2} /(\mathrm{V} \mathrm{s})$ and it did not depend on implantation dose. This could be explained by assuming that the implanted donors and compensating acceptors create donor-acceptor pairs which do not scatter electrons as efficiently as single ions do [2]. The pairs could be created during recrystallization when sample was annealed at $600^{\circ} \mathrm{C}$.

Time resolved photoluminescence [4] was applied to determine photoexcited carrier lifetime. Ti:sapphire laser (pulse duration $100 \mathrm{fs}$ ) was used for excitation of electron-hole pairs at the band edge region. The photoexcited carrier density was about $10^{18} \mathrm{~cm}^{-3}$. The photoluminescence (PL) decay was of a single exponential type over about two orders of magnitude. Figure $1 \mathrm{~b}$ presents the photocarrier lifetime dependence on the implantation dose for the $600^{\circ} \mathrm{C}$ annealed samples implanted with the four species studied. The values of PL decay times for GaAs implanted at a certain dose with $\mathrm{As}, \mathrm{Ga}$ and $\mathrm{O}$ were very close to each other. They decreased monotonically from $7 \mathrm{ps}\left(10^{14} \mathrm{~cm}^{-2}\right.$ dose $)$ down to $2 \mathrm{ps}\left(10^{16} \mathrm{~cm}^{-2}\right.$ dose). The samples implanted with silicon exhibited slightly longer PL decay times changing from $11 \mathrm{ps}\left(10^{14} \mathrm{~cm}^{-2}\right.$ dose $)$ down to $3.4 \mathrm{ps}\left(10^{16} \mathrm{~cm}^{-2}\right.$ dose $)$ and $0.4 \mathrm{ps}$ $\left(5 \times 10^{16} \mathrm{~cm}^{-2}\right.$ dose $)$.
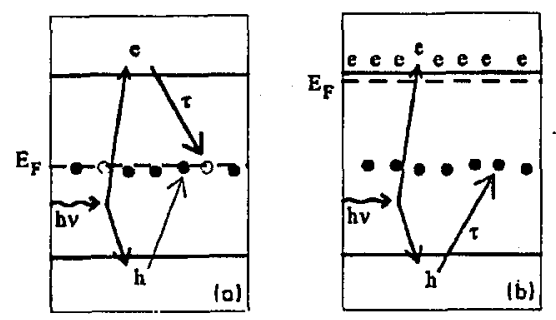

Fig. 2. The scheme of carrier trapping in GaAs samples (a) implanted with As, Ga or $\mathrm{O}$ and (b) implanted with Si ions.

The observed PL decay time was much shorter than the carrier recombination time of a standard $\mathrm{GaAs}$, therefore some extra recombination process had to be present in the implanted samples. PL excitation depth for the light used was much smaller than $1 \mu \mathrm{m}$. From TEM studies we know that this layer is free of precipitates. Moreover, there were no metallic precipitates at all in $\mathrm{GaAs}: \mathrm{O}$ and GaAs:Si, therefore the fast trapping had to occur due to large number of point defects. It is important to compare $n$-type ( $\mathrm{Si}$ implanted) and semi-insulating (As, Ga, O implanted) samples (see Fig. 2). In the semi-insulating material (Fig. 2a) PL decay was determined by electron trapping while in the $n$-type material (Fig. 2b) minority carriers (holes) trapping was decisive. The best electron trap is an ionized donor. It could be EL2 in semi-insulating material and Si in $n$-type material. The most efficient hole traps are ionized acceptors and judging from our experimental data their concentration should be nearly the same as donors. Analysis of PL decay time (see Fig. 1b) show that the trapping process in As, Ga 
and $\mathrm{O}$ implanted samples was nearly twice faster than in Si implanted samples, but in both cases the dependence of time vs. dose was very similar. It seems that some correlation between these two processes should exist. The best explanation of this phenomenon is that the recombination takes place at the donor-acceptor pairs. Such pairs could have high cross-section for both electron and hole trapping. The cross-sections can differ by a factor of about 2 for hole and electron capture, leading to different trapping times, but capture rates of electrons and holes should be proportional. This is what we observe in our experiment.

In summary, the dependence of carrier trapping times on the implantation dose for GaAs implanted with various ions at $\mathrm{MeV}$ energies and thermally annealed at $600^{\circ} \mathrm{C}$ is presented. We distinguish two types of samples. First, semi-insulating material with trapping times slightly shorter and second, $n$-type (strongly compensated) material with decay times longer (but still in the ps range). We postulate that in both materials fast recombination of carriers could be explained by capture at donor-acceptor pairs.

We acknowledge the partial support of this work by an Australian Bilateral Exchange Research Grant.

\section{References}

[1] F. Smith, A.R. Calawa, C.L. Chen, M.J. Mantra, L.J. Mahoney, IEEE Electron. Device Lett. 9, 77 (1988).

[2] K.P. Korona, Acta Phys. Pol. A 88, 463 (1995).

[3] A. Krotkus, S. Marcinkevicius, J. Jasinski, M. Kaminska, H.II. Tan, C. Jagadish, Appl. Phys. Lett. 66, 3304 (1995).

[4] A. Krotkus, S. Marcinkevicius, V. Pasiskevicius, U. Olin, Semicond. Sci. Technol. 9, 1382 (1994). 